This item was submitted to Loughborough's Institutional Repository (https://dspace.lboro.ac.uk/) by the author and is made available under the following Creative Commons Licence conditions.

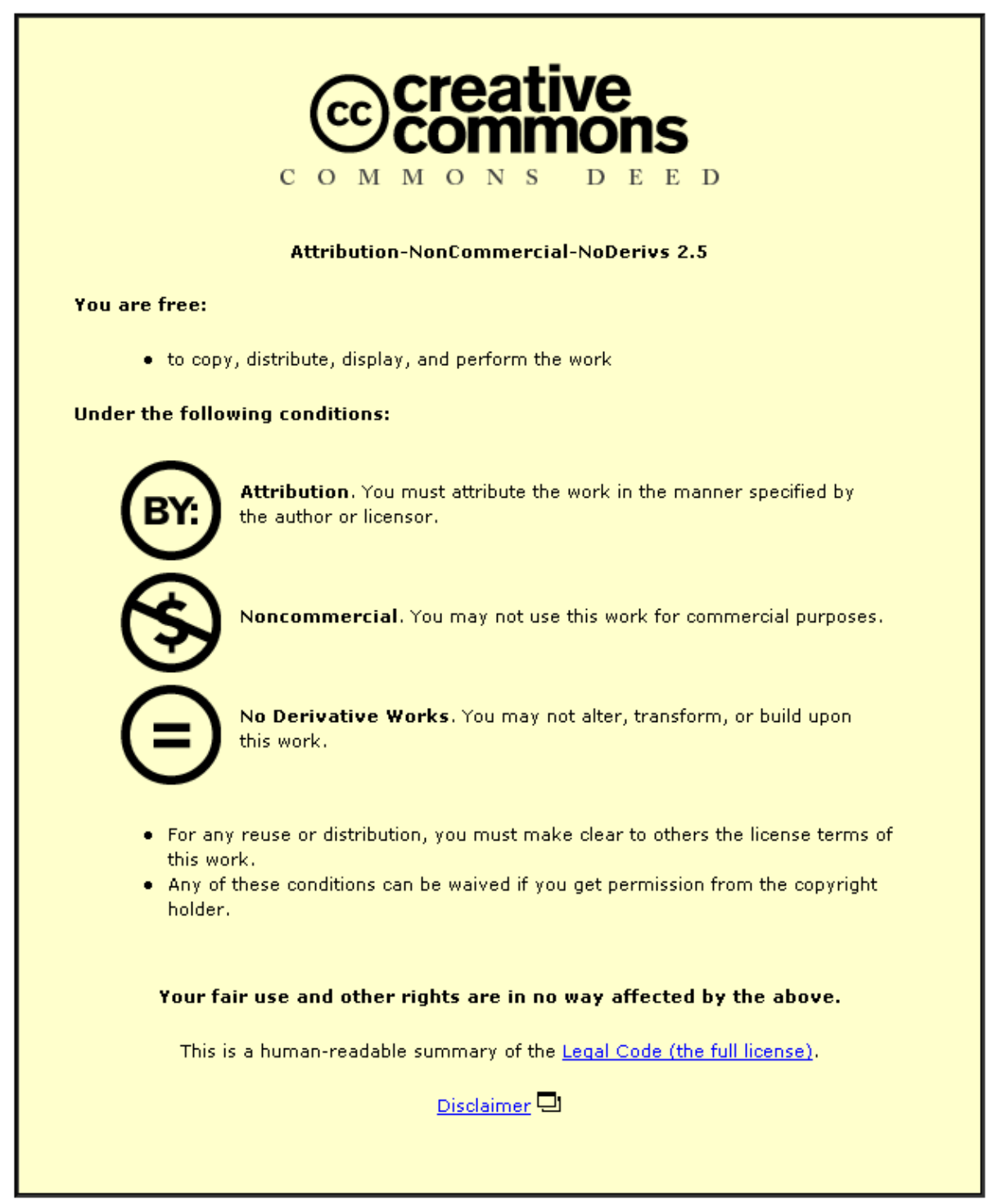

For the full text of this licence, please go to: http://creativecommons.org/licenses/by-nc-nd/2.5/ 


\title{
Numerical Analyses of Bubble Point Tests used for Membrane Characterisation: Model Development and Experimental Validation
}

\author{
V. Nassehi ${ }^{*}$, D.B. Das, I.M.T.A. Shigidi and R.J. Wakeman \\ Department of Chemical Engineering, Loughborough University, Loughborough, \\ Leicestershire LE11 3TU, UK
}

\begin{abstract}
A numerical model for simulating the microhydrodynamics inside different pore sizes was developed in this work, using a continuous penalty finite element scheme. This scheme combines the flexibility in modelling two phase systems, as the one simulated in this work with accuracy. The volume of fluid (VOF) method was applied to track the motion of the gasliquid interfacial boundary as an approach to monitor the repulsion of the wetting liquid from the pores to detect their bubble pressures. To resolve the complexities arising from the inclusion of the surface tension at the liquid-gas interface as an unknown dynamic condition it is treated as a resistance force in the equations of motion. The effects of the surface tension and other forces such as the buoyancy are then determined by model calibration with respect to a set of experimental data. To obtain the experimental data, the bubble point test was used to characterise different Nuclepore track etched membrane samples, which provided insights into the mechanisms underlying the test and into the interpretation of the pore size distribution. The experimental data are used to calibrate the numerical model. The calibrated model was, in turn, used to predict the outcome of bubble point tests for a range of inlet boundary conditions. The results obtained from these simulations are shown to be in good agreement with of the experimental data, indicating the ability of the developed model to accurately predict the bubble point pressure.
\end{abstract}

Keywords: Membrane Characterisation; Bubble Point Test; Finite Element Method; Penalty Scheme; Volume of Fluid; Microhydrodynamics.

\footnotetext{
${ }^{*}$ Corresponding author. Tel.: +44 (0)1509222522; fax: +441509223923

E-mail address: v.nassehi@lboro.ac.uk
} 


\section{INTRODUCTION}

Membrane is one of the most widely used means of physical separations and has broad industrial applications. Traditionally, the main objective of applying the membranes has been either clarification of liquor or separation of particles for recovery or others. More recently, membranes have been also used in tissue engineering for variety of purposes, e.g., to support cell/tissue growth in bioreactors, screening of cells and molecules, etc.

To obtain high efficiency, the membranes should have the ability to separate a wide size distribution of particles, offer appropriate hydraulic resistance to the filtrate flow and, if necessary, allow easy discharge of accumulated solids from its surface. Also, it should be resistant to chemical attack and swelling when in contact with filtrate and show good heat resistance within the temperature ranges of the filtration process, in addition to having sufficient strength to withstand filtering pressure and mechanical wear. These characteristics point to the need to understand the importance of comprehensively characterising the morphology of the membranes and their potential capability to retain the dispersed phase, and why this knowledge is vital in the design and selection of the appropriate membranes to maximise the efficiency of the processes involved.. Many methods can be applied to characterise a membrane, e.g., mercury porosimetry, scanning electron microscopy (SEM), and others (Mulder, 2003). All the methods are generally dependent on different physical and/or chemical phenomena. Each of these tests provides limited knowledge about certain parameters; combining the results gained from different methods presents a broad and more in-depth understanding about the media properties.

Measurement of the bubble point test is one of the characterisation methods, which can also act as a test of monitoring membrane integrity (Adham et al, 1998; Mulder, 2003). MiettonPeuchot et al (1997) and Hirt and Nichols (1981) were able to use the same procedure to measure the pore size distribution for both clean and fouled membranes and thus were able to define a methodology for choosing membranes appropriate to microfiltration applications for the use of recycling alkaline cleaning solutions in dairy products industry. Calvo et al (1995) and Hernandez et al (1996) studied the surface of several track etched microporous polycarbonate membranes using the bubble point test to define the pore size distribution and have combined their results with SEM pictures as an additional supporting method. The bubble point test has been used to characterise stainless steel membranes (Ames et al, 2003), ceramic membranes (Jakobs and Koros, 1997), woven cloths (Rideal et al, 2004), and non- 
woven nanofibre filter media (Jena and Gupta, 2004), tissue engineering membranes/scaffold (Safina et al., 2005), etc, showing that the test can be applied in a wide range of applications after appropriate experimental protocols and settings have been established. Jena and Gupta (2005) further extended the methodology to characterise the pore structure of complete filter cartridges.

The primary flow mechanism that occurs inside the membrane pores during the bubble point test involves drainage of a liquid (wetting phase) by gas (non-wetting phase). This two-phase flow is characterised by the movement of the gas/liquid interfaces, determined by a number of factors such as surface tension at the gas/liquid interfaces. Dynamics of the gas/liquid interfaces have been studied in different context by many researchers. For example, Polynkin et al (2004) considered liquid displacement by investigating two scenarios: a) maintaining a steady state gas penetration velocity along the cylindrical tube, and b) a constant gas pressure applied at the inlet. Their main concern was to examine the inertial effects on the two cases and to relate the shape of the bubble developed inside the cylindrical tube to the Reynolds number. In their research conducted at a macro scale the surface tension, which is a prime factor within the bubble point test, was excluded from the governing equations. Taha and Cui (2002) studied the gas liquid interfacial effect on cross flow operations in ultra-filtration by monitoring the injection of the gas phase into the feed and applied the volume of fluid (VOF) technique to detect the interfacial boundaries between the two phases. They used a commercial software (FLUENT) to carry out their simulations and obtained an improvement in the permeate flux by introducing the gas bubbles into the filtration process and were able to predict the shape of the bubbles inside a tubular membrane with reasonable accuracy.

However, it seems there is a lack of numerical tool to analyse the bubble point test, the dynamics of the gas/liquid interfaces inside the membrane pores during the test, and, hence the morphology of the membrane. In this paper, we present a general, in-house approach to address these issues. We consider the effects of the surface tension of the wetting liquid and introduce it into the working equations; different types of boundary conditions were investigated to determine conditions which are the most representative of the experimental results.We use in house data from the bubble point test on Nuclepore track etched membrane to develop and validate the numerical tool. Nuclepore track etched membranes have well defined pore structures (Figure 1), have almost cylindrical parallel pores and are used in many applications including biological engineering (e.g., separation of cells). However, the 
developed numerical tool is a general tool and can be applied to analyse the characteristics of other membranes including tissue engineering membranes/scaffold. Furthermore, please note we only apply the methodology to study the movement of gas/liquid interfaces inside membranes pores. But, the methodology may be extended to study the dynamics of liquidliquid interfaces within membranes, in necessary. The purpose of the present paper is therefore simply to present the developed model and demonstrate its validity for simple membranes (e.g., Nuclepore track etched membranes).

EXPERIMENTALS PROCEDURE As mentioned earlier the main purpose of conducting our in house experiments on bubble point test was to validate the numerical model developed in this work. In this section, we present the experimental procedures and some results briefly.

In general, the bubble point tests rely on imposing an incrementally increasing pressure on a membrane sample wetted by a low surface tension liquid, such as alcohol, Porofil, Galwick or Silwick. This wetting liquid then spontaneously fills the pores of the sample. The increase of pressure difference across the sample during a test results in a gradual expulsion of the wetting liquid from the largest to the narrowest filled pores resulting in a flow throughout the membrane (Meltzer, 1987; Mayer, 2002; Reichelt, 1987). Air is passed through the membrane to drain the liquid out of the sample. The flow of air is monitored by a sensitive flow transducer. The pressure required to obtain this flow is recorded and the diameter of the pore is calculated using a form of Young-Laplace equation as presented in equation (1), before incrementing the pressure until the relation between the applied pressure and the flow rate becomes linear, which indicates that all the pores within the sample are now emptied. This is known as the wet curve. The shape of the wet curve obtained provides an insight into the pore size distribution within the tested sample. It was noticed that the expulsion of the wetting liquid generates a residual liquid layer in the pore but it has no impact on the pore diameter calculations.

$\Delta \mathrm{P}=\frac{4 \sigma \cos \omega}{\mathrm{d}}$

where $\Delta P$ being the applied pressure, $d$ is the pore diameter, $\sigma$ is the surface tension of the wetting liquid and $\omega$ is its contact angle with the solid matrix of the membrane.

A dry curve is obtained by applying the same technique on a dried sample and, the intersection between the two curves is usually at the pressure required to dry the minimum 
pore size within the tested sample. A half dry curve is determined from the values obtained from the dry curve, and is obtained using half of the flow rate through the dry sample.

During this work a PMI Porometer was used to measure the pore size distributions and, to verify the results. Some of the measurements were repeated using a Coulter II Porometer. In both cases the membrane pore size distributions were indirectly measured using the gas flow detected after evacuating each group of pores to calculate the fraction of a pore diameter from gas flow differential data using equation (2):

$\%$ of pores with size $d=100 \times\left[\left(\frac{F_{w, i+1}-F_{w, i-1}}{F_{d}}\right)\left(\frac{1}{2}\right)\right]$

where $F$ is the wet (subscript $w$ ) and dry (subscript $d$ ) flow at time $i$.

The theory behind the process is that the ratio of an incremental change in the gas flow through a wetted membrane to the total gas flow through the dry membrane (over the increment of $\Delta P_{i-1}$ to $\left.\Delta P_{i+1}\right)$ can be used to estimate the fraction of the total pores with a specific diameter at $\Delta P_{i}$ by using Young-Laplace equation (Farahbakhsh and Smith, 2004; Martinez-Diez et al, 2000).

Nuclepore track etched polycarbonate membranes manufactured by Whatman international Ltd were used in this work with different nominal mean pore sizes ranging from 0.2 to $12 \mu \mathrm{m}$. These membranes (Figure 1) have a low porosity of about $5 \%$ and the pores are defined to be cylindrical through pores, i.e. there are no blind or closed pores. The wetting liquid used in this work was Galwick (supplied by PMI) with surface tension of 15.9 dyne/cm. A PMI capillary flow porometer was used to analyse the samples.

As an example of the results, those for a $1 \mu \mathrm{m}$ sample will be presented in what follows. Figure 2 shows the wet, dry and half dry curves. Initially the applied pressure has no impact on the wet curve until the pore with the largest diameter starts to drain at pressure of about 40 $\mathrm{kPa}$. The sharp increase of the flow rate after the bubble point is a result of the narrow pore size distribution of the tested sample. Increasing the pressure results in detecting more narrower pores till the sample is completely dried at $60 \mathrm{kPa}$ and the relation between the pressure and the flow rate becomes linear. At this point the wet curve ends and the dry test starts. The intersection of the wet and dry curves occurs after the location of the minimum pore within the tested sample. The minimum pore diameter detected for the nominal $1 \mu \mathrm{m}$ 
sample was $0.26 \mu \mathrm{m}$. The half dry curve in Figure 2 shows a mean pore size detected at pressure of $46 \mathrm{kPa}$.

The pore size distributions measured for the track etched membranes is very narrow, as shown in Figure 3. The measured mean pore sizes were found to be close to the manufactures specification. For example, in the case of the $1 \mu \mathrm{m}$ sample, $93 \%$ of the pores had a diameter of $0.97 \mu \mathrm{m}$. Table 1 presents the summary of a range of pore sizes measured. For all data presented in this work, the results are the average of 5 tested samples and the manufacturers' nominal rating is used as the identifier of the samples.

The presence of multiple pores generated from double and sometimes triple overlapped pores causes the detection of pore sizes beyond the range specified by the manufacturers. This can also be seen in the SEM in Figure 1.

\section{MATHEMATICAL MODEL}

In the modelling techniques described in this paper, the finite element method (FEM) is used to discretize the fluid flow equations in conjunction with the volume of fluid (VOF) technique to keep track of the gas/liquid interfaces in the pores. The FEM is chosen due to its flexibility in dealing with complex geometries, whereas the VOF technique provides a convenient method to relocate the interfacial boundary between the gas and the wetting liquid after the fluid velocity field is known. This method has proven to be both effective and convenient in solving two phase flow problems (Nassehi, 2002).

The numerical modelling of the flow is based on the fundamental governing equations that reflect the fluid dynamical characteristics of the regime. Due to the very low Reynolds number of the gas-liquid flow inside pores, the resulting fluid flow is described by the creeping incompressible Stokes equation.

For an isothermal transient flow in a two dimensional Eulerian framework the conservation of mass is written as:

$$
\frac{\partial v_{x}}{\partial x}+\frac{\partial v_{y}}{\partial y}=0
$$

where $v_{x}, v_{y}$ are the $x$ and $y$ components of the velocity vector in a planar coordinate system. 
The components of the equation of motion (representing conservation of momentum) for creeping $(\operatorname{Re}<1)$ flow of Newtonian fluids are described in terms of Stokes equation as:

$$
\begin{aligned}
& \rho \frac{\partial v_{x}}{\partial t}=-\frac{\partial p}{\partial x}+\mu\left(\frac{\partial^{2} v_{x}}{\partial x^{2}}+\frac{\partial^{2} v_{x}}{\partial y^{2}}\right)+\rho f_{x}+R_{x} \\
& \rho \frac{\partial v_{y}}{\partial t}=-\frac{\partial p}{\partial y}+\mu\left(\frac{\partial^{2} v_{y}}{\partial x^{2}}+\frac{\partial^{2} v_{y}}{\partial y^{2}}\right)+\rho f_{y}+R_{y}
\end{aligned}
$$

where $\rho$ is the fluid density, $\mu$ is the viscosity, $p$ is the pressure, $f_{x}$ and $f_{y}$ are the components of body forces such as buoyancy and gravity, and $R_{x}$ and $R_{y}$ are the components of the surface tension which is included as an additional resistance force affecting the two-phase flow regime.

The advancing flow front of the gas within a pore is simulated using the VOF technique as mentioned earlier. In a fixed two dimensional Cartesian coordinate system the flow front position is traced by a variable $0 \leq F \leq 1$. The substantial time derivative of this variable is:

$$
\frac{D F}{D t}=\frac{\partial F}{\partial t}+v_{x} \frac{\partial F}{\partial x}+v_{y} \frac{\partial F}{\partial y}=0
$$

In a two phase system separated by an interface, the variable $F$ in equation (5) is a probability density function taken to be zero in all locations where, say liquid (or phase one) is the only existing phase. Therefore in locations where purely gas (phase two) is present its value is 1 . Along the interface separating the phases the value of $F$ is between 0 and 1 . Starting from an initial configuration (e.g. $F=1$ every where which may mean in the present case as completely gas filled pores) the solution of equation (5) in conjunction with the model equations provides a simple method for updating the position of phases and hence the interface boundary between the gas and liquid phases (Nassehi, 2002; Das and Nassehi, 2004). In this work we have used a version of the VOF technique in which the moving boundary regime is considered as a two phase flow regime where the filled pores (wetting liquid) and the empty pores (gas) sections are considered as different phases. This is similar to the method developed by Hirt and Nichols (1981) and Jeong and Yang (1998).

The flow model is solved for the entire domain with the correct physical properties introduced into each region. A decoupled solution of equation (5) with the flow model generates the 
values of $F$. These values range between 0 and 1 . The values of $F$ are interpreted in the following way: for a generated value of $0 \leq F<0.3, F$ is considered to be 0 ; for $0.3 \leq F<0.75, F$ is taken as 0.5 ; and for $F \geq 0.75, F$ is then taken as 1 , where 1 signifies that an element is filled with gas and 0 indicates that it is filled by the wetting liquid (Nassehi, 2002). A value of $F=0.5$ indicates the location of the interfacial boundary separating the two phases. Hence in the context of the present work it represents the displacement of the wetting liquid by the compressed gas. Values of physical parameters of each phase in the flow field are related to the position of this interfacial boundary using the following equation:

$\chi=\chi_{l} F+\chi_{g}(1-F)$

where $\chi$ is a given physical parameter (e.g. density) and $\chi_{l}$ and $\chi_{g}$ refer to the wetting liquid and gas values of this parameter, respectively. As is evident from the above explanations in the VOF technique a change in the density of the fluid continuum occurs at the interface between the phases. Therefore the buoyancy resulting from this effect should be taken into account. This force appears as a body force in the equation of motion.

\section{Formulation of the weighted residual statement}

Substitution of the field variables (i.e., velocity components and pressure) in the equation of motion by approximate forms (indicated by $\widetilde{v}_{i}$ and $\tilde{p}$ )results in the generation of residuals. These residuals are weighted by appropriate functions and integrated over the solution domain to disappear. Therefore:

$$
\int_{\Omega} W_{j} \rho \frac{\partial \widetilde{v}_{i}}{\partial t} d \Omega-\int_{\Omega} W_{j} \mu \nabla^{2} \widetilde{v}_{i} d \Omega+\int_{\Omega} W_{j} \nabla \widetilde{p} d \Omega=\int_{\Gamma} W_{j} R_{i} d \Gamma+\int_{\Omega} W_{j} \rho f_{i} d \Omega
$$

$$
i=x, y
$$

where $W_{j}$ are the weight functions $(j=x, y), \Omega$ is the solution domain and $\Gamma$ represents the interface boundary between the liquid and gas phases in the pores.

The surface tension acting on the interface between the two phases is given as:

$R_{i}=-n_{i} 2 H \sigma$

Where $n_{i}$ is the component of unit vector normal to the interface (i.e. $\underline{n}$ ), $H$ is the interface curvature and $\sigma$ is the surface tension coefficient (a physical parameter depending on the properties of the fluid and surface) . Using Frenet's first formula (Olson and Kock, 1994): 


$$
\frac{d \underline{t}}{d s}=2 H \underline{n}
$$

where $\underline{t}$ is the tangent vector to the interface surface. Therefore the surface tension term in the weighted residual statement (7) is written as:

$$
\int_{\Gamma} W_{j} 2 H \sigma \underline{n} d \Gamma=\int_{s_{1}}^{s_{2}} W_{j} 2 H \sigma \underline{n} d s=\int_{s_{1}}^{s_{2}} W_{j} \frac{d \underline{t}}{d s} \sigma d s=-\int_{s_{1}}^{s_{2}} \sigma \frac{d W_{j}}{d s} \underline{t} d s+\sigma\left[W_{j} \underline{t}\right]_{1}-\sigma\left[W_{j} t\right]_{2}
$$

where $s$ is the arc length along the interface curve.

From equation (10) we get:

$$
\frac{d W_{j}}{d s}=W_{j, x} \cdot t_{x}+W_{j, y} \cdot t_{y}
$$

where

$$
\left\{\begin{array}{l}
t_{x}=\frac{d x}{d s} \\
t_{y}=\frac{d y}{d s}
\end{array}\right.
$$

The load vector appearing on the right hand side of the weighted residual statement representing the surface tension is hence derived as

$$
R_{i}=-\int_{s_{0}}^{s_{1}} \sigma \frac{d}{d s} W_{j} t d s+\sigma\left[W_{j} t\right]_{1}-\sigma\left[W_{j} t\right]_{2}
$$

The direct use of equation (13) in a computational scheme presents significant difficulties and can only be attempted under very restrictive simplifying conditions. This is because that at the edges of the interface the tangential terms in the right hand side of equation (13) should be written in terms of the contact angle $\omega$ as:

$T=\sigma \cos \omega$

The contact angle depends on the physical properties of the wetting liquid and surface of contact and ideally it should be measured experimentally. In addition in an Eulerian frame work (as is the case in the present study) the line integral representing the first term in the right hand side of equation (13) should be calculated in each element by determining the points of intersection of the interface curve with element boundaries corresponding to $F=0.5$. Therefore, for example, the $x$ component of this term is found as:

$$
\int_{\Gamma} \sigma t_{x} \frac{d W_{j}}{d s} d \Gamma=\sum_{q=1}^{m} \sigma\left(\frac{\partial W_{j}}{\partial x}(\xi, \zeta)^{q} \cdot t_{\xi}^{q}+\frac{\partial W_{j}}{\partial y}(\xi, \zeta)^{q} \cdot t_{\zeta}^{q}\right) \Delta s_{q}
$$


where $(\xi, \zeta)$ represent a local elemental coordinate system, $q$ is an index identifying a particular element side and $m$ is the total number of element sides, $t_{\xi}^{q}$ and $t_{\zeta}^{q}$ are local components of $t_{x}$. The arc length $\left(\Delta s_{q}\right)$ should be calculated in terms of the global coordinates of the points of intersections of the interface curve with the sides of each element as:

$$
\Delta s_{q}=\sqrt{\left(x_{2}^{q}-x_{1}^{q}\right)^{2}+\left(y_{2}^{q}-y_{1}^{q}\right)^{2}}
$$

Derivatives of the weight function originally given in terms of global $(x, y)$ coordinates should also be transformed into the local elemental system using the Jacobian of coordinate transformation. As the geometry of the interface is expected to change continuously with the flow deterministic calculation of the surface tension force through equation (16) is not possible. Furthermore under general conditions a priori determination of the points of intersection of the interface boundary will also be impossible. Iterative techniques based on an initial assumption and repetitive solutions can be attempted. However, there is no proof that such a computationally expensive procedure will converge at all or if it converges it will provide a physically realistic result. In addition, physical factors which affect the surface coefficient, in general, and the contact angle along the edges of the interface cannot be easily measured. To avoid these problems, in the present work, a single set of experimental results is used to adjust the value of the surface tension term in the right hand side of the original weighted residual statement (equation 7) so that the model results fit the experimental data. After this initial calibration the fitted value of the surface tension is stored and kept constant for subsequent computations for which the model predictions are compared with the experimental data. Effects of other forces such as the buoyancy and gravity can also be determined during this calibration. Thus, the present bubble point test combined with the described model calibration can be viewed as a method for the determination of the surface tension for any given membrane. The outlined methodology provides a very convenient way of resolving the almost impossible problem of the inclusion of a realistic surface tension in the model simulations. Furthermore one should bear in mind that, although, a totally independent experimental measurement of the surface tension potentially provides a general predictive mathematical model the measurement should be repeated for each membrane as the physical conditions will be different in each case. Therefore, despite significantly high computational cost and effort a universally applicable predictive model can only yield 
meaningful results for only a single type of membrane after inserting a set of uniquely measured parameters. In contrast in the present work using a single set of easily measurable experimental results (i.e. bubble point test) surface tension for any given type of porous membrane can be calibrated. This data can then be used to predict the performance of the membrane under a wide variety of conditions without resorting to time consuming experiments.

\section{Solution Methodology}

The weighted residual equation (7) involves both pressure and velocity components as the field unknowns. Therefore to make the system of equations determinate they should be augmented by a similar weighted residual statement derived using the continuity equation. The divergence free equation representing the mass balance in an incompressible flow system does not include a pressure term. Therefore its numerical solution in conjunction with the equation of motion presents a classical stability problem. In order to resolve this difficulty the numerical scheme used should satisfy a stability condition known as the LadyzhenskajaBabuška-Brezzi (LBB) condition (Reddy, 1993). In the present work we have used the continuous penalty scheme which, in addition to fulfilling the LBB condition, results in a compact form of the working equations, hence minimizing computational costs. The continuous penalty formulation eliminates the pressure as an unknown variable through relating it to the incompressibility constraints as:

$p=-\lambda\left(\frac{\partial v_{x}}{\partial x}+\frac{\partial v_{y}}{\partial y}\right)$

where $\lambda$ is a penalty parameter represented as the product of a very large dimensionless number and fluid viscosity (Nassehi, 2002):

$\lambda=\lambda^{*} \mu$

where $\mu$ is the fluid viscosity and $\lambda^{*}$ is a large positive number determined by numerical experiments conducted to examine the stability of the solution. Inclusion of fluid viscosity in the penalty parameter is a necessary condition to make equation (17) dimensionally consistent. After the substitution of pressure in the components of equation of motion via the 
penalty formulation the solution domain is discretized into a number of sub-domains of finite size (finite elements) as $\sum_{e=1}^{E} \Omega_{e}=\Omega$, where $E$ is the total number of elements in the computational mesh. Following the normal finite element procedures within each element components of velocity, which are now the remaining field unknowns, are replaced by approximations in terms of interpolation functions and their corresponding nodal values as:

$$
\begin{aligned}
& \widetilde{v}_{x}=\sum_{l=1}^{r} v_{x}^{l}(t) N_{l}(x, y), \\
& \widetilde{v}_{y}=\sum_{l=1}^{r} v_{y}^{l}(t) N_{l}(x, y)
\end{aligned}
$$

where $v_{x}^{l}(t)$ and $v_{y}^{l}(t)$ are nodal values of the components of the velocity vector at node number $l$ of a given element $\Omega_{e}$ as function of time, $\gamma$ is the total number of nodes per element and $N_{l}$ is the interpolation function associated with node number $l$. Using the standard Galerkin method in which the weight functions in the weighted residual statement are identical to the interpolation functions the elemental working equations of the present model are derived in a matrix form as:

$$
\left[\begin{array}{ll}
M_{j l} & 0 \\
0 & M_{j l}^{22}
\end{array}\right]\left\{\begin{array}{l}
\dot{v}_{x} \\
\dot{v}_{y}
\end{array}\right\}+\left[\begin{array}{ll}
K^{11} & K^{12} \\
K^{21} & K^{22}
\end{array}\right]\left\{\begin{array}{c}
v_{x} \\
v_{y}
\end{array}\right\}=\left\{\begin{array}{c}
B_{j}^{1} \\
B_{j}^{2}
\end{array}\right\}
$$

where

$$
M_{j l}^{11}=M_{j l}^{22}=\int_{\Omega e} \rho N_{j} N_{l} d x d y
$$

and

$$
K_{i j}^{11}=\int_{\Omega_{e}}\left[(\lambda+2 \mu)\left(\frac{\partial N_{i}}{\partial x} \frac{\partial N_{j}}{\partial x}\right)+\mu \frac{\partial N_{i}}{\partial y} \frac{\partial N_{j}}{\partial y}\right] d x d y
$$

etc. (Nassehi, 2002). As already explained in the continuous penalty method used here the pressure is removed from the working equations. However, in order to impose the pressure as the inlet boundary condition it needs to be maintained explicitly in the load vector. Therefore the right hand side terms in equation (21) are written, for example, as:

$$
B_{j}^{1}=\int_{\Gamma_{e}} N_{i}\left[\left(\lambda p+2 \mu \frac{\partial u^{e}}{\partial x}\right) n_{x}+\mu p n_{y}+g_{x}\right] d \Gamma_{e}
$$

In equation (24) $u^{e}$ is an elementally interpolated value of velocity and $g_{x}$ represents the effects of surface tension and other body forces (e.g., buoyancy). The time derivatives of the 
velocity components in equation (21) are treated using the implicit $\theta$ method. Taking $t_{\theta}$ to be a time level between $t_{n}$ and $t_{n+1}$ the system of equations (21) is written as:

$[M]\{\dot{X}\}_{\theta}+[K]\{X\}_{\theta}=[\{B\}]_{\theta}$

where the subscript $\theta$ indicates that the weighted residual statement is derived using a value for $\theta$ between 0 and 1, therefore:

$\{\dot{X}\}=\frac{\{X\}_{n+1}-\{X\}_{n}}{\Delta t},\{X\}_{\theta}=(1-\theta)\{X\}_{n}+\theta\{X\}_{n+1}$, and $\{B\}_{\theta}=(1-\theta)\{B\}_{n}+\theta\{B\}_{n+1}$

After the time stepping the final working equation of the present scheme is obtained as:

$$
\left.\left.\left.\left.\left\{\mid M_{i j}\right\rfloor+\theta \Delta t \mid K_{i j}\right\rfloor_{n+1}\right\}\{X\}_{n+1}-\left\{\mid M_{i j}\right\rfloor-(1-\theta) \Delta t \mid K_{i j}\right\rfloor_{n}\right\}\{X\}_{n}=\left[(1-\theta)\left\{B_{j}\right\}_{n}+\theta\left\{B_{j}\right\}_{n+1}\right\rfloor \Delta t
$$

Working equation (27) is used to obtain the velocity field in the gas/fluid system.

The pressure field is found using the following variational recovery method. Using the penalty formulation,

$$
\int_{\Omega_{e}} w p d \Omega_{e}=-\int_{\Omega_{e}} w \lambda\left(\frac{\partial v_{x}}{\partial x}+\frac{\partial v_{y}}{\partial y}\right) \Omega_{e}
$$

The discretization of equation (28) using the Galerkin finite element yields a scheme for the calculation of nodal pressures as:

$$
\int_{\Omega_{e}} N_{I} \sum N_{K} p_{K} d \Omega_{e}=\stackrel{\lambda}{*} \int_{\Omega_{e}} N_{I} \mu\left(\frac{\partial v_{x}}{\partial x}+\frac{\partial v_{y}}{\partial y}\right) d \Omega_{e}
$$

where the penalty parameter in equation (28) is substituted using equation (18). The coefficient matrix on the left-hand side of equation (29) is the mass matrix given by:

$$
M_{I K}=\int_{\Omega_{e}} N_{I} N_{K} d \Omega_{e}
$$

This matrix is diagonalized using a simple mass lumping technique to minimize the computational cost of the pressure calculations. After the computation of the velocity field the r.h.s. of equation (29) is determined and hence the pressure can be found. The important point to note that the integration of r.h.s in equation (29) should be based on a reduced quadrature (Nassehi, 2002). 


\section{Assumptions and Boundary conditions}

The following assumptions were made to reduce the complexity of the problem being analysed:

1. The pores of Nuclepore tack etched membranes being simulated were cylindrical, thus the tortuosity factor is 1 .

2. The amount of gas absorbed by the wetting liquid is negligible, hence solubility is not included within the model.

3. No diffusion occurs on the walls of the pores by either the air or the wetting liquid.

4. The air compressibility, and hence change in air density, is negligible within the pores. The value of the inlet pressure boundary condition is increased manually at the end of each simulation until the bubble point is reached.

The effects of slip and no-slip boundary conditions imposed on the pore walls were investigated. Imposition of no slip velocity conditions at the solid wall is based on the assumption that the shear stress at these surfaces always remains below a critical value to allow a complete wetting of the wall by the fluid. The second type of boundary condition, the slip condition, occurs when the shear stress exceeds the threshold causing the fluid to move over the surface of the wall and was introduced by directly incorporating the slip condition into the working equations using the following forms:

$$
\begin{aligned}
& v_{x}-v_{x b}=-\mu \beta n_{y}\left[2\left(\frac{\partial v_{x}}{\partial x}-\frac{\partial v_{y}}{\partial y}\right) n_{x} n_{y}+\left(\frac{\partial v_{x}}{\partial y}-\frac{\partial v_{y}}{\partial x}\right)\left(n_{y}^{2}-n_{x}^{2}\right)\right] \\
& v_{y}-v_{y b}=-\mu \beta n_{x}\left[2\left(\frac{\partial v_{x}}{\partial x}-\frac{\partial v_{y}}{\partial y}\right) n_{x} n_{y}+\left(\frac{\partial v_{x}}{\partial y}-\frac{\partial v_{y}}{\partial x}\right)\left(n_{y}^{2}-n_{x}^{2}\right)\right]
\end{aligned}
$$

where $v_{x b}$ and $v_{y b}$ are the components of solid wall velocity (in the present case the pore walls are stationary and hence these are equal to 0 ) the slip coefficient $\beta$ is defined as $\beta=\frac{\beta_{0}}{l} . \beta_{0}$ is the initial slip coefficient and $l$ is a characteristic flow domain dimension. The limits of $\beta$ are 0 and $\infty$. These limits correspond to no slip conditions where $\left(v_{x}=v_{x b}, v_{y}=v_{y b}\right)$ and complete wetting of the walls simultaneously. In cases where partial slip occurs, the value of slip coefficient must be found using experimental measurements. As discussed later in this paper 
in this study the perfect slip case is used as a numerical experiment to prove the validity of the adopted solution scheme.

\section{Solution Algorithm}

The computer code constructed to carry out the present simulations is based on the following algorithm.

Step 1- The domain under investigation is discretized into a mesh of finite elements.

Step 2- Initial configurations representing the filled domain are considered and an array consisting of the appropriate values of $F=0$ along the filled domain and $F=1$ at the inlet nodes where the applied air that is introduced after the initial values for nodal pressure are imposed, obtained from the experimental settings. An array containing the non-slip boundary conditions along the external boundaries of the investigated domain is set up and stored.

Step 3- The time variable is updated by incrementing it by $\Delta t$.

Step 4- Initial boundary conditions are used to solve the flow and distribution function equations to obtain the velocity distribution and then by using the Variational Recovery Method to calculate the pressure along the tested domain (Ghoreishy and Nassehi, 1997).

Step 5- New values of the material properties, density and viscosity, are determined from the computed velocity field by solving equation (5).

Step 6- Steps 4 and 5 are repeated until convergence occurs before using the velocity field to solve the surface position function.

Step 7- Using the updated values of the surface position function, the location of the interfacial boundary between the two phases is identified showing the penetration of the applied gas into the filled domain.

Step 8- When the final time is reached the calculations terminate, otherwise steps 3-8 are repeated and the solution progresses with time. 


\section{Physical Data}

The physical data used in the solution of the governing equations are: wetting liquid density $=$ $1830 \mathrm{~kg} \mathrm{~m}^{-3}$; wetting liquid viscosity $=0.0372 \mathrm{~Pa} \mathrm{~s}$; density of air $=1.2929 \mathrm{~kg} \mathrm{~m}^{-3}$; viscosity of air $=0.251 \times 10^{-4} \mathrm{~Pa} \mathrm{~s}$. The penalty parameter $\lambda^{*}=7.5 \times 10^{11}$, time increment $\Delta t=0.025 \mathrm{~s}$, and the time stepping parameter $\theta=0.75$. The flow domain is discretized into nine nodded biquadratic finite elements and the convergence of the solution is checked via mesh refinement by increasing the number of elements after the first simulation.

\section{SIMULATION RESULTS AND DISCUSSIONS}

Results obtained for two different solution domains are presented in this paper. The first domain consists of pore diameters 1, 2, 3, 4 and $6 \mu \mathrm{m}$ (Figure 4). The length of the pores was assumed to be the same as the thickness of the membrane samples, and an extra length was added at the inlet to initialise the model calculations. The ability of the model to predict the bubble point for a membrane having a number of pores with different diameters was tested. The applied pressure required to detect each pore diameter was recorded. This investigation monitored the gas liquid displacement and also provided knowledge about both velocity and pressure profiles which helped understand the microhydrodynamics occurring inside the pores.

These are a multiple pores domain where a number of pores with a range of diameters between $1 \mu \mathrm{m}$ and $6 \mu \mathrm{m}$ are connected together at the inlet (Figures 5) and another consisting of a single $1 \mu \mathrm{m}$ pore. The length of the pores is $10 \mu \mathrm{m}$ in each case. The microhydrodynamics occurring inside both domains were studied using the developed model. A comparison between the results obtained experimentally and numerically was carried to validate the effectiveness of the model in detecting the bubble point for different pore diameters.

Starting from an inlet pressure of $1 \mathrm{kPa}$, the pressure was incremented until a displacement of the air-liquid interface into a pore was obtained. This occurred at about $10 \mathrm{kPa}$ with the slip boundary conditions applied on the pore walls. Some results generated from this boundary condition are presented in Figure 5. These results, obtained after $1 \mathrm{~s}, 3.75 \mathrm{~s}$ and $6.35 \mathrm{~s}$, show 
the gas liquid displacement inside the domain and how the gas moves in a plug flow fashion regardless of the pore diameter. This is indicative of the failure of the slip conditions to model the pressure flow (Poiseuille flow) occurring inside the pores which leads to failure in detecting the bubble point within the tested domain. The simulated output showing a uniform rise of the liquid in pores of different diameters, which is expected under perfect slip conditions, provides a strong indication of the model accuracy.

To overcome these difficulties in the model, non-slip boundary conditions were introduced at the pore walls. A typical velocity profile is shown in the Figure 6, indicating the direction of the fluid flow from the inlet (the bottom of the diagram) towards the exit of the domain. The magnitudes associated with the profiles in the pores in Figure 6 indicate that the velocity of the fluid increases with the pore diameter, as would be expected due to the same pressure difference existing across the length of all the pores. The diagram also shows the development of parabolic profile in each pore, even though the velocity distribution at the entrance to the pores is asymmetric due to the presence of flow in neighbouring pores.

The velocity profiles infer that the amount of fluid passing thorough a single pore is related to its diameter, as would be expected. The non-slip condition leads to a zero velocity of the wetting fluid at the pore boundaries (the resolution of the diagram in Figure 6 prevents this from being shown) which suggests the presence of a thin film sticking at the pore walls (which is apparent in Figure 7). This is consistent with what has been observed in many studies, e.g., the deliquoring of filter cakes and other porous media, where liquid is retained by the pore structure and is only subsequently removed by evaporative mechanisms. The same situation prevails experimentally in porometry, although with membranes the duration of the evaporation period is short due to their thinness. Similar findings were also reported by Polynkin et al (2004).

Figure 7 shows the penetration of the gas into the filled pores after different time periods with a pressure of $10 \mathrm{kPa}$ applied at the inlet. The surface tracking function (F, equation (5)) is used to locate the surface front; the approximations described following equation (5) (assuming $F=0$ when the generated value of $0 \leq F<0.3, F=0.5$ when $0.3 \leq F<0.75$, and $F=1$ when $F \geq 0.75$ ) cause the low resolution, and hence the lack of smoothness, of the profile of the gas-liquid interface shown when $t>0$. 
Figure 7 shows the development of the air penetration into the pores at an inlet pressure of 10 $\mathrm{kPa}$ after 1, 3.75 and 6.25 seconds. Air breakthrough at the outlet can be observed and complete displacement of the wetting liquid is obtained from the pore except in the region close to the pore walls where the thin film is retained. Hence, the bubble point for the membrane has been passed after 6.25 seconds at an applied pressure of $10 \mathrm{kPa}$. Whilst the results show the penetration of the applied gas into the pore at different pore sizes, it is clear that with the $6 \mu \mathrm{m}$ pore diameter air breakthrough is reached before that of $4 \mu \mathrm{m}$ diameter, and even before the gas has penetrated into the $1 \mu \mathrm{m}$ pore. This suggests that the pressure applied at the inlet was not sufficient to detect the bubble point for a $1 \mu \mathrm{m}$ pore when it exists within the pore size distribution shown in Figure 7.

Numerical simulations were also carried out for a single sized pore (it was assumed that this would be representative of a truly monosized pore size distribution). For a single $1 \mu \mathrm{m}$ pore, an inlet pressure was increased incrementally up to $10 \mathrm{kPa}$, the no-slip boundary conditions were specified on the walls of the pore, and the results showed no displacement of the wetting liquid. At a pressure of $30 \mathrm{kPa}$, air breakthrough occurred and displacement of the wetting liquid was obtained throughout the pore; breakthrough occurred after 3.125 seconds. Figure 8 shows the progress of the gas penetration as the air-liquid interface moves through the pore after $0.375,1.5,2.5$ and 3.125 seconds. The imposition of the non-slip boundary conditions on the wall again resulted in the presence of a film of the wetting liquid along the pore walls. Further, gas penetration rate into the pore accelerated during the displacement process; for example, in the case of the single pore shown on Figure 8 the air had penetrated into about $6 \%$ of the pore length in $0.375 \mathrm{~s}$, but after $1.5 \mathrm{~s}$ the penetration had reached about $55 \%$.

The velocity profile for displacement from the single $1 \mu \mathrm{m}$ pore is shown in Figure 9, indicating the establishment of a parabolic profile a short distance into the pore. This is in agreement with predictions by previous researchers (Reddy, 1993; Zienkiewicz and Taylor, 2000). After entrance effects, the pressure profile in Figure 9 shows a constant pressure gradient along the pore length.

The aim of the model development was to calculate bubble point pressures for membranes with different sizes of pores. Porometers were used to measure the bubble point pressure and pore size distributions. These instruments measure the pressure at which air flow breaks through the porous material and thereafter the relationship between air flow rate and air 
pressure as more liquid is expelled from the medium. This information is then used to calculate the pore sizes of the membrane. Since the pressure at which an air flow through the porous body is initiated is used to calculate the bubble point, for consistency with those experimental apparatuses the same is used in the numerical calculations here. Strictly the bubble point pressure is the minimum pressure needed to initiate displacement of the wetting fluid, that is, the pressure at which air first enters a pore (Bear, 1975). An implicit assumption is therefore made in this work (and in the analysis of data from porometers) that the breakthrough pressure is equal to the bubble point pressure; this is not strictly true but one is a good approximation of the other when the porous material is very thin, which is the case with the membranes used in this study.

The developed model does not include all factors that affect either bubble point or breakthrough pressures; for example, surface wettability, contact angle and surface tension are important factors to consider when working with membrane materials, as is any form of heterogeneity in the pore structure. In the case of the membranes used in the experiments here, the main heterogeneity arises from the existence of pore coincidence (usually as doublets or triplets) and from curvatures at the pore ends. The pores are not therefore perfect cylinders as assumed in the model. These several factors will cause deviations between the calculated and experimental breakthrough pressures.

To compare the results obtained from the porometry experiments with those generated from the model, a factor (herein referred to as a calibration factor) was introduced into the equations (31) and (32). The calibration factor was found to play a significant role on the results obtained. Several values of this factor were examined numerically, each yielding a pressure-diameter curve of similar form but displaced on the pressure axis as shown on Figure 10. For comparison, the data obtained from the porometer experiments is also presented on Figure 10. From this it is seen that a calibration factor value of 75 gives good agreement between the numerical computations and the experimental data. The experimental and simulated breakthrough pressures for different pore diameters are summarised on Table 2. It is recognised that the "best" calibration factor value is likely to be dependent on the hydrophobicity/ hydrophilicity of the membrane and the tortuousity of the pores, and that at the moment it is not known how specific the factor is to the particular type of membranes used in this study. 


\section{CONCLUSIONS}

The bubble point was measured for a range of tack etched membranes with different nominal pore size ratings. These data gave baseline information for the development of a mathematical model to simulate membrane testing in a porometer. The model was developed using the finite element method with a penalty scheme to study the microhydrodynamics inside a single pore. The adopted penalty scheme provides flexibility in the imposition of inlet pressure boundary conditions reflecting the way in which porometers operate, as well as the automatic satisfaction of the stability condition required in the modelling of divergence free (incompressible flow). The model was used to study the microhydrodynamics occurring inside single pores and a distribution of pore sizes. The volume of fluid (VOF) method was incorporated into the model to study the movement of the air-liquid interfacial boundary. This enabled the bubble point (or breakthrough pressure) for the pores to be calculated numerically, and the results were compared with the experimental data. The general forms of the experimental and computed relationships between bubble point pressure and pore diameter agreed well, and a calibration factor enabled agreement of the breakthrough pressures to within $10 \%$.

The penetration of the applied air at the inlet of the pores was simulated with time for multiple pores, where a number of pores with a wide range of diameters were connected at the inlet. The microhydrodynamics were presented showing how the velocity profile inside each pore is developed. The interfacial boundary between the gas and the liquid has also been studied and it was found that liquid in pores with the larger diameters is displaced sooner than that in smaller diameter pores. This penetration of air into differing sized pores at different pressures led to simultaneous flow in the different pores, with air breakthrough at the outlet face occurring for the larger pores earlier than for the smaller ones.

The results obtained from the experimental porometer settings were compared with those generated from the mathematical model. The calibration factor was used to minimize the error between the results obtained experimentally and those generated by the model. Use of different values for the calibration factor led to the same shape of curve describing the relationship between the breakthrough pressure and the pore diameter, but the factor had the effect of displacing the curve up/down on the pressure axis. A value of 75 was found to give a good fit of the model to the experimental data, and the results demonstrated the capability of the model to simulate the bubble point for different pore diameters. 


\section{References}

Adham S., Gagliardo P., Smith D., Ross D., Gramith K. and Trussell R. (1998) Monitoring the integrity of reverse osmosis membranes, Desalination, 119, 143-150.

Ames R.L., Bluhm E.A., Douglas Way J., Bunge A.L., Abney K.D. and Schreiber S.B. (2003) Physical characterization of $0.5 \mu \mathrm{m}$ cut-off sintered stainless steel membranes, Journal of Membrane Science, 213, 13-23.

Bear J. (1975) Dynamics of Fluids in Porous Media, American Elsevier, New York.

Calvo J.I., Hernandez A., Caruana G. and Martinez L. (1995) Pore size distributions in microporous membranes: I. Surface study of track-etched filters by image analysis, Journal of Colloid and Interface Science, 175, 138-150.

Das, D.B. and Nassehi, V. (2004). Validity of a moving boundary finite element model for salt intrusion in a branching estuary", Advances in Water Resources, 27(7), 1st July 2004, 725-735, DOI: 10.1016/j.advwatres.2004.04.001

Farahbakhsh K. and Smith D.W. (2004) Estimating air diffusion contribution to pressure decay during membrane integrity tests, Journal of Membrane Science, 237, 203-212.

Ghoreishy M.H.R. and Nassehi V. (1997) Modeling the transient flow of rubber compounds in the dispersive section of an internal mixer with slip-stick boundary conditions, Advances in Polymer Technology, 16, 45-68.

Hernandez A., Calvo J.I., Pradanos P. and Tejerina F. (1996) Pore size distributions in microporous membranes. A critical analysis of the bubble point extended method, Journal of Membrane Science, 112, 1-12.

Hirt C.W. and Nichols B.D. (1981) Volume of fluid (VOF) method for the dynamics of free boundaries, Journal of Computational Physics, 39, 201-225.

Jakobs E. and Koros W.J. (1997) Ceramic membrane characterization via the bubble point technique, Journal of Membrane Science, 124, 149-159.

Jena A. and Gupta K. (2004)Characterisation of nanofibre filtration media by capillary flow porometry, Filtration, 4(3), 176-180.

Jena A. and Gupta K. (2005) Characterization of the pore structure of complete filter cartridges using high flow porometry, Filtration, 5(3), 183-187.

Jeong J.H. and Yang D.Y. (1998) Finite element analysis of transient fluid flow with free surface using VOF (Volume Of Fluid) method and adaptive grid, International Journal for Numerical Methods in Fluids, 26, 1127-1154.

Martinez-Diez L., Florido-Diaz F.J. and Vazquez-Gonzalez M.I. (2000) Characterization of hydrophobic microporous membranes from water permeation, Separation Science and Technology, 35, 1377-1390.

Mayer E. (2002) Porometry characterization of filter media, Proc. American Filtration and Separation Society $15^{\text {th }}$ Annual Technical Conference \& Expo, American Filtration and Separations Society, Galveston, Texas.

Meltzer T.H. (1987) Filtration in the Pharmaceutical Industry, Marcel Dekker, New York.

Mietton-Peuchot M., Condat C. and Courtois T. (1997) Use of gas-liquid porometry measurements for selection of microfiltration membranes, Journal of Membrane Science, 133, 73-82.

Mulder, M (2003). Basic Principles of Membrane Technology. Kluwer Academic Publishers, Dordrecht.

Nassehi V. (2002) Practical Aspects of Finite Element Modelling of Polymer Processing, Wiley, Chichester, West Sussex, England ; New York, NY.

Olson, L and Kock, E. (1994) A variational approach for modelling surface tension effects in inviscid fluids, Computational Mechanics, 14, 140-153. 
Polynkin A., Pittman J.F.T. and Sienz J. (2004) Gas displacing liquids from tubes: high capillary number flow of a power law liquid including inertia effects, Chemical Engineering Science, 59, 2969-2982.

Reddy J.N. (1993) An Introduction to the Finite Element Method, McGraw-Hill, New York.

Reichelt G. (1987) Bubble point measurements on large areas of microporous membranes, Journal of Membrane Science, 60, 253-259.

Rideal G., Mayer E. and Lydon R. (2004) Comparative methods for the pore size calibration of filter media, Filtration, 4(1), 29-33.

Safinia L, Blaker JJ, Maquet V, Boccaccini AR, Mantalaris A, Bismarck A (2005). Characterisation of 'wet' polymer surfaces for tissue engineering applications: Are flat surfaces a suitable model for complex structures? E-POLYMERS , Article Number: 010Taha T. and Cui Z.F. (2002) CFD modelling of gas-sparged ultrafiltration in tubular membranes, Journal of Membrane Science, 210, 13-27.

Zienkiewicz O.C. and Taylor R.L. (2000) The Finite Element Method, ButterworthHeinemann, Oxford ; Boston. 


\begin{tabular}{|c|c|c|c|}
\hline $\begin{array}{c}\text { Manufacturers' } \\
\text { nominal rating } \\
(\mu \mathrm{m})\end{array}$ & $\begin{array}{c}\text { Bubble point pore } \\
\text { diameter } \\
(\mu \mathrm{m})\end{array}$ & $\begin{array}{c}\text { Mean flow pore } \\
\text { diameter } \\
(\mu \mathrm{m})\end{array}$ & $\begin{array}{c}\text { Minimum flow } \\
\text { pore diameter } \\
(\mu \mathrm{m})\end{array}$ \\
\hline 0.2 & 0.308 & 0.242 & 0.15 \\
\hline 0.8 & 1.15 & 1.146 & 0.666 \\
\hline 1 & 1.746 & 0.97 & 0.505 \\
\hline 5 & 7.148 & 5.817 & 1.048 \\
\hline 12 & 18.496 & 15.301 & 2.834 \\
\hline
\end{tabular}

Table 1. Samples of pore diameters for different track etched membranes used in the experiments. 


\begin{tabular}{|l|c|c|c|c|c|}
\hline \multirow{2}{*}{} & \multicolumn{5}{|c|}{ Pore diameter $(\mu \mathrm{m})$} \\
\cline { 2 - 6 } & 1 & 3 & 5 & 7 & 12 \\
\hline Experimental breakthrough pressure $(\mathrm{kPa})$ & 64.13 & 21.02 & 13.0 & 9.1 & 5.34 \\
\hline Simulated breakthrough pressure $(\mathrm{kPa})$ & 65.0 & 21.0 & 12.0 & 10.0 & 5.0 \\
\hline $\begin{array}{l}\text { Deviation of simulated values from } \\
\text { experimental values (\%) }\end{array}$ & 1.4 & 0.1 & 7.7 & 9.9 & 6.4 \\
\hline
\end{tabular}

Table 2. Breakthrough pressures related to pore diameters using a calibration factor of 75 .

Show where in equation is calibration factor? 


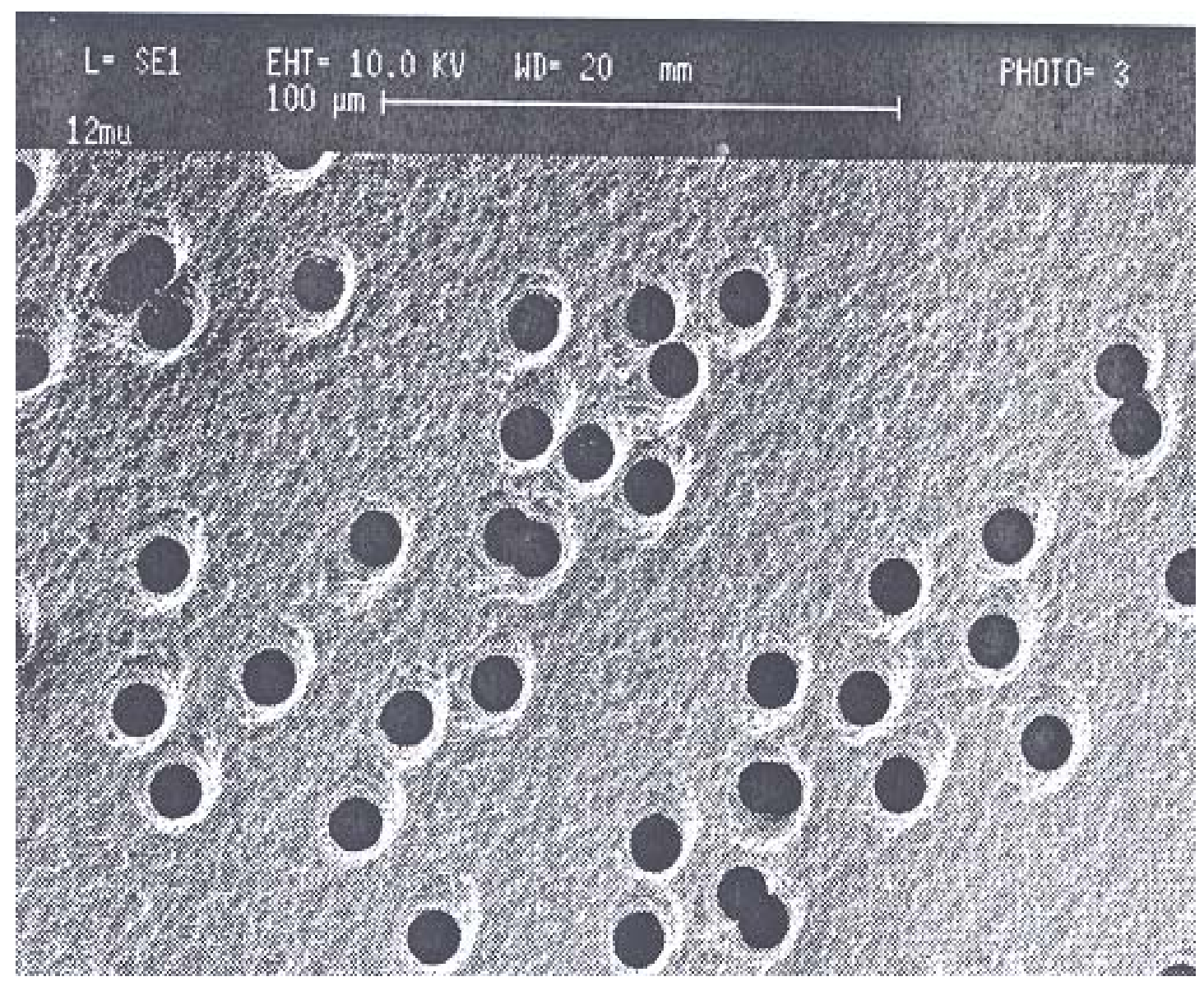

Figure 1. Scanning electron microscope (SEM) picture of a Nuclepore track etched membrane. 


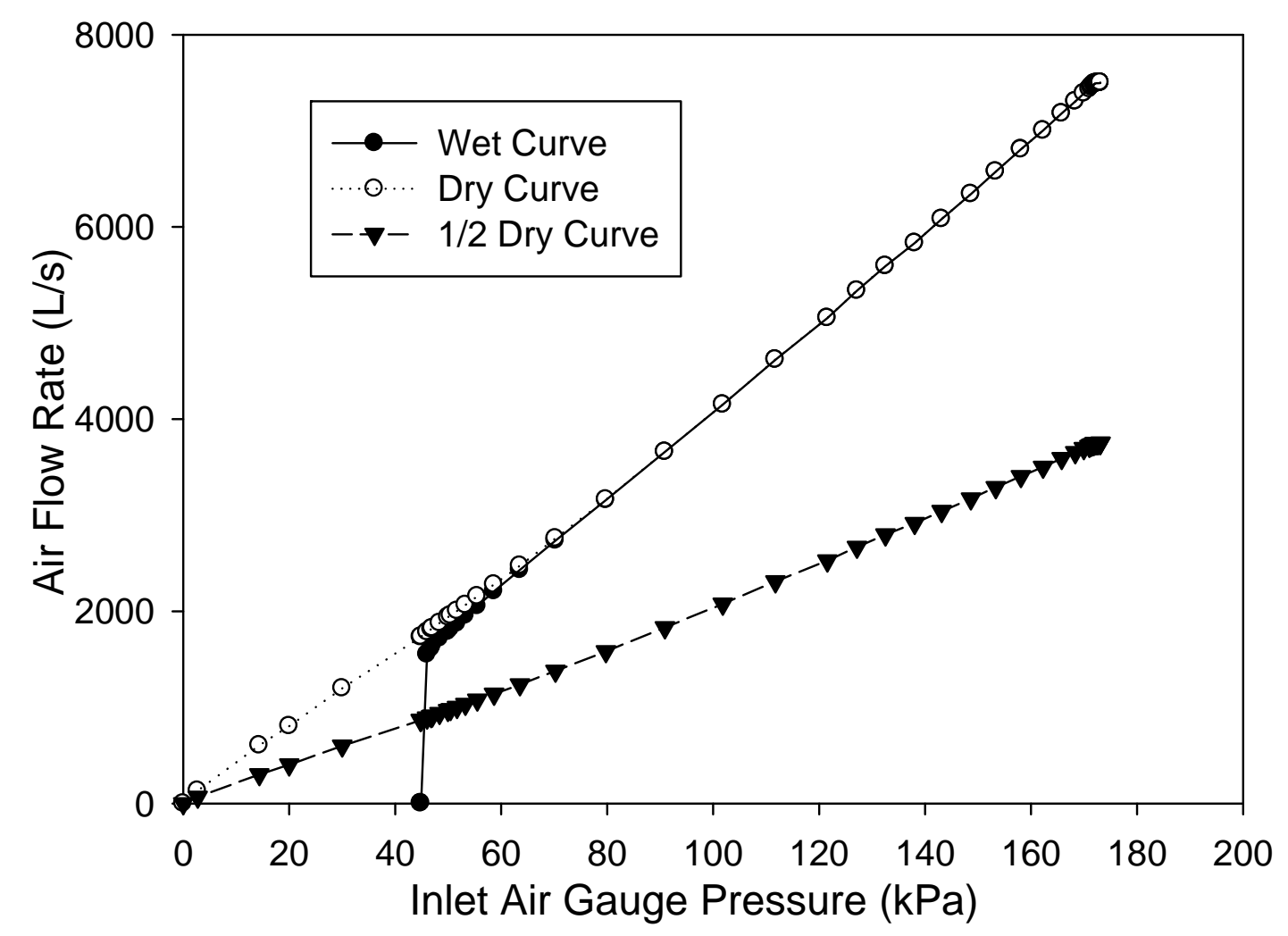

Figure 2. Wet, dry and half dry flow rate $v s$. applied pressure curves for a nominal $1 \mu \mathrm{m}$ membrane. 


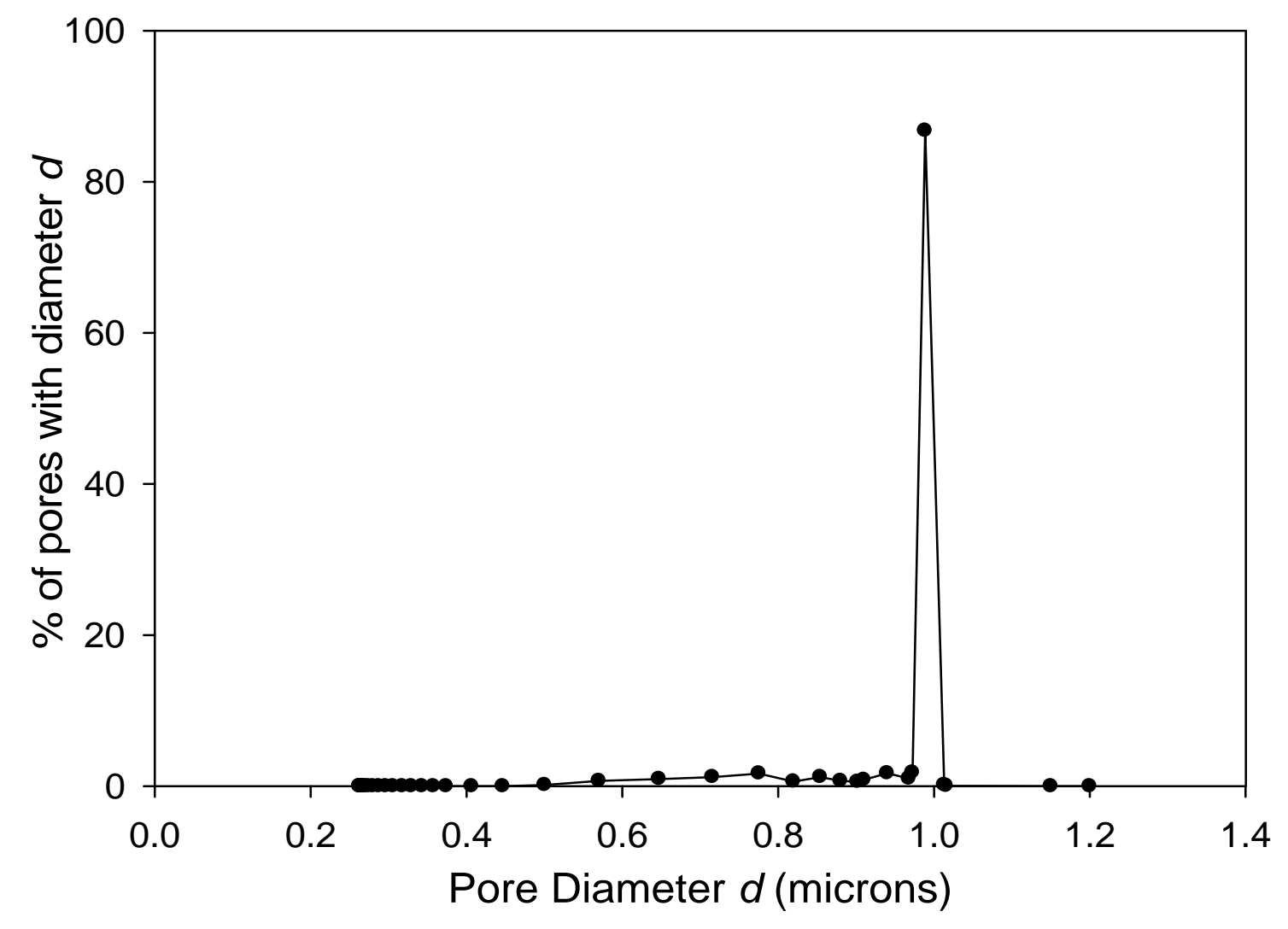

Figure 3. Pore size distribution for the nominal $1 \mu \mathrm{m}$ sample. 


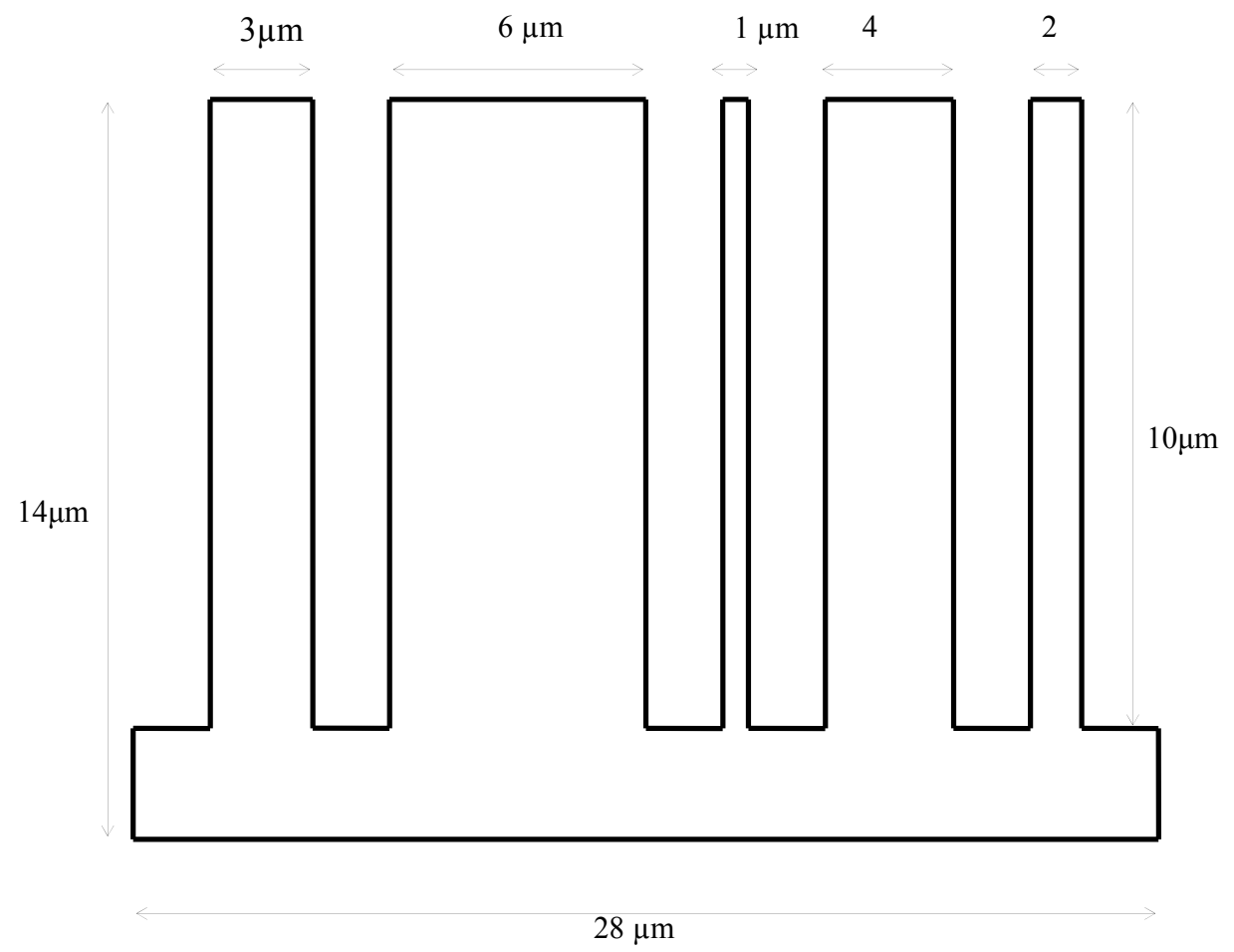

Figure 4. Dimensions for multi-pore domain. 


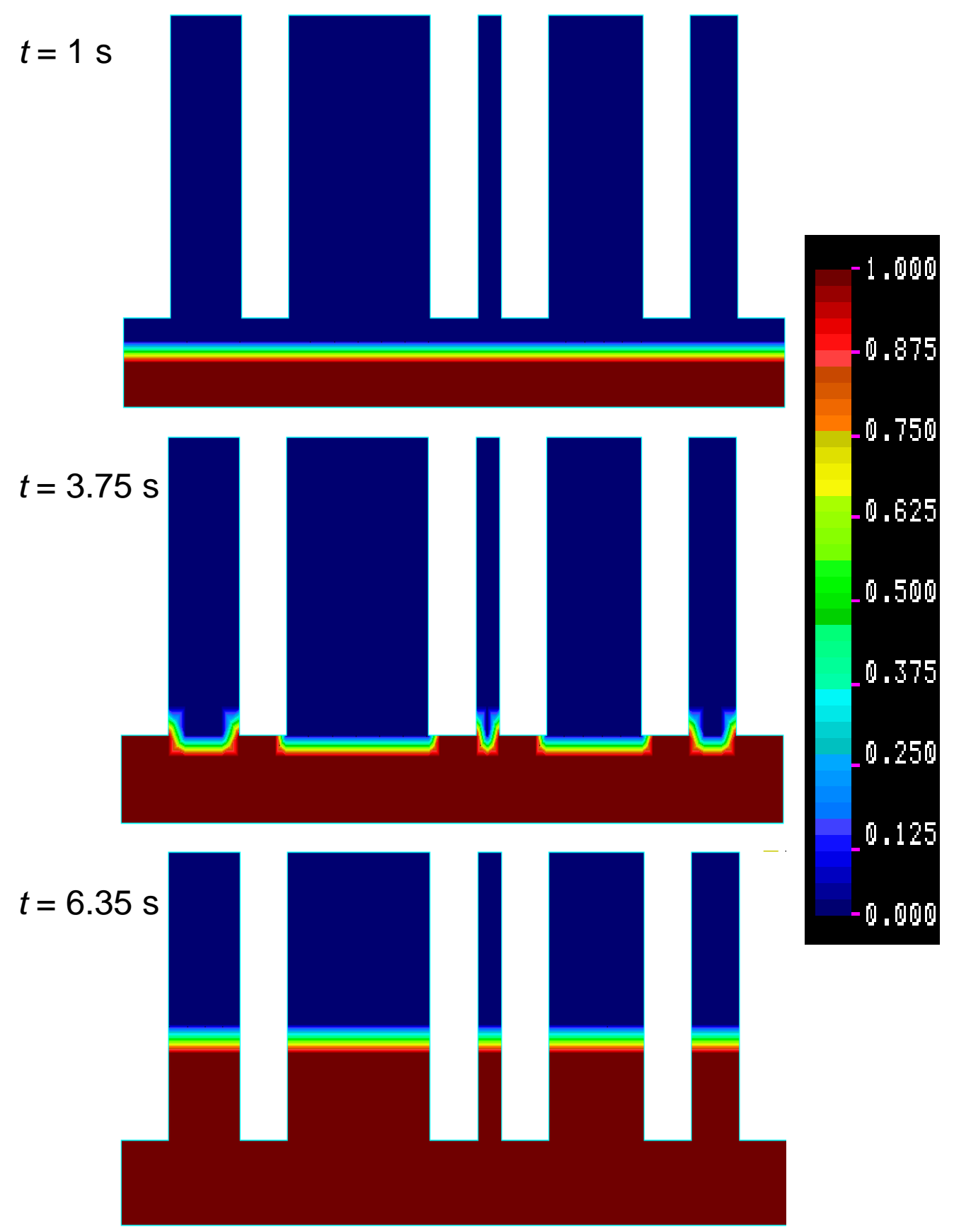

Figure 5. Pressure scale profiles showing the air-liquid interfacial boundaries at different time steps for the multi-pore domain with slip boundary conditions with an inlet pressure of $10 \mathrm{kPa}$ (dimension of the domains are shown in Figure 4) 


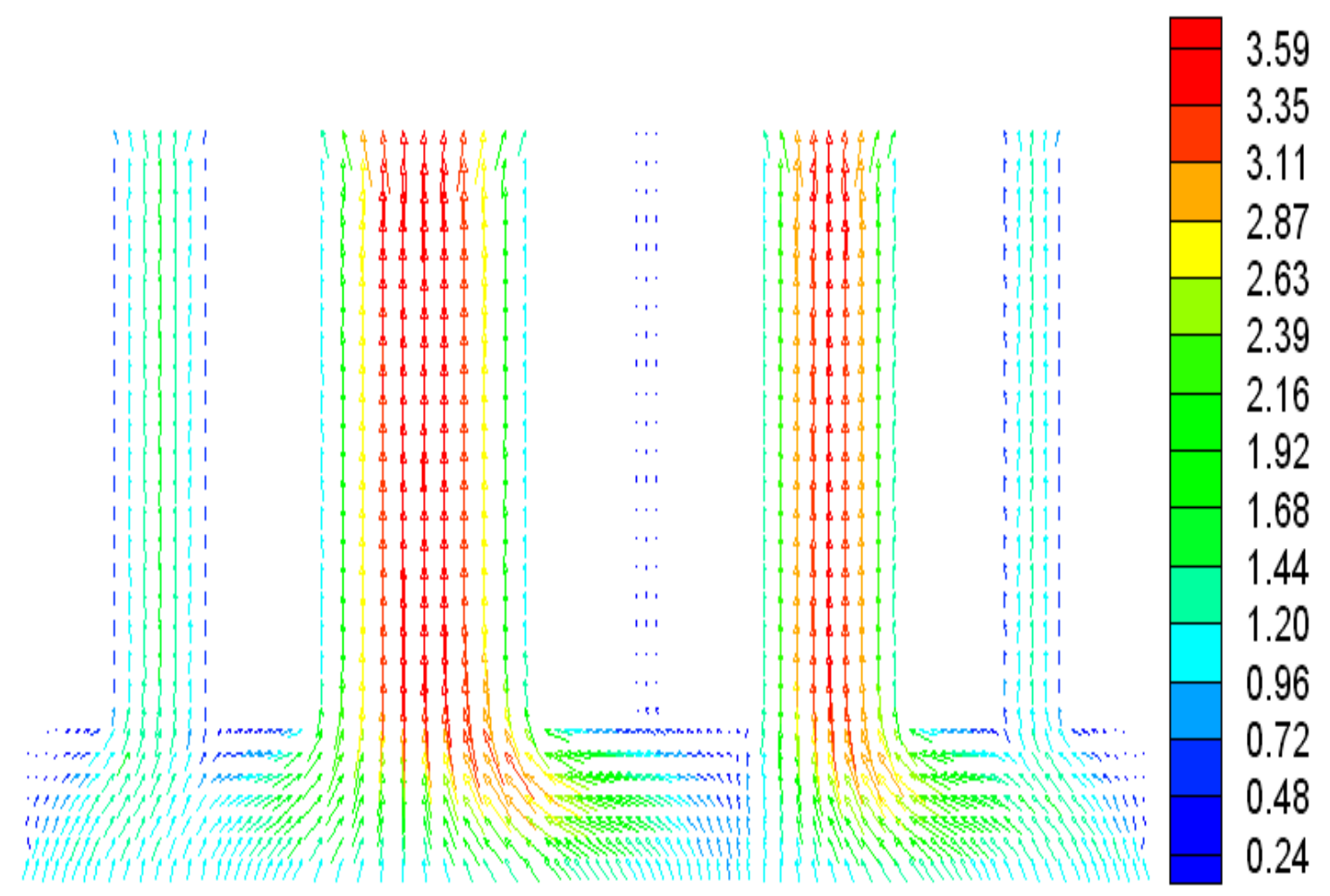

Figure 6. Simulated flow field in the multi-pore domain with a non-slip condition at steady state, $0.125 \mathrm{~s}$ after applying a pressure of $10 \mathrm{kPa}$ at the inlet (dimension of the domains are shown in Figure 4).

Unit of scale? 

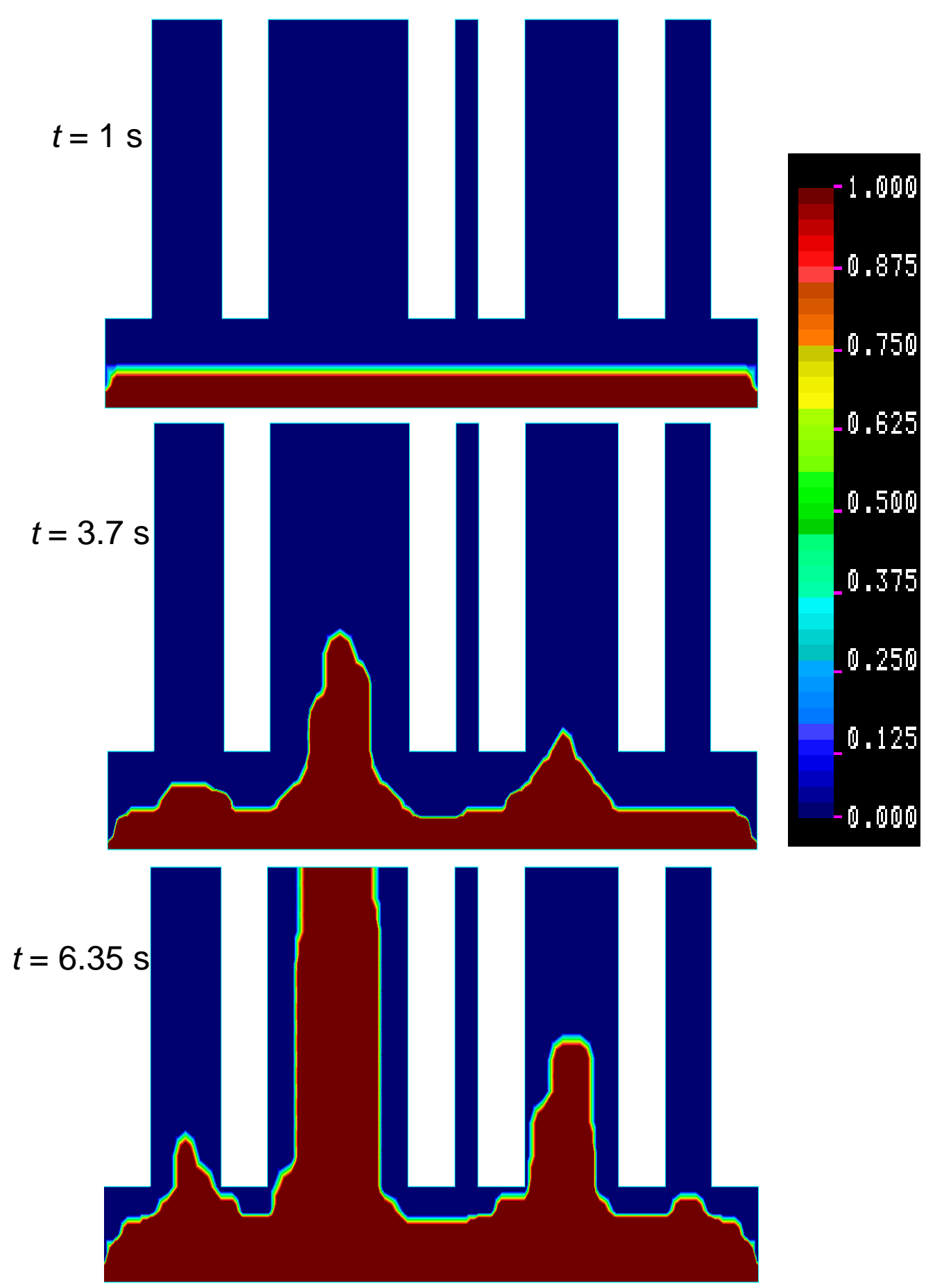

Figure 7. Pressure profiles showing the air-liquid interfacial boundaries at different time steps for the multi-pore domain with non-slip boundary conditions with an inlet pressure of 10 $\mathrm{kPa}$ (dimension of the domains are shown in Figure 4).

Unit of scale 


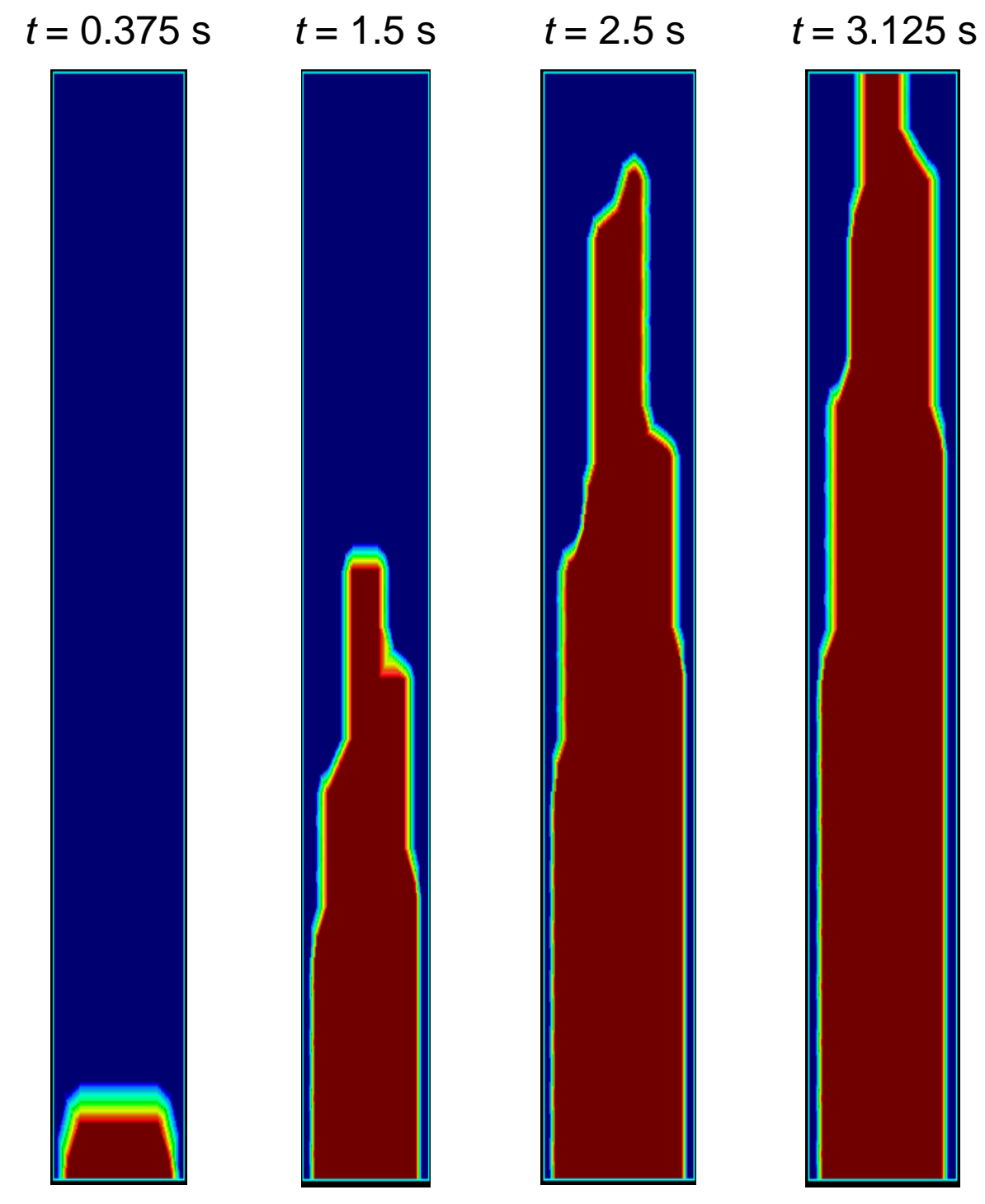

Figure 8. The air-liquid interface at different times for a $1 \mu \mathrm{m}$ diameter pore with an inlet pressure of $30 \mathrm{kPa}$ and a non-slip boundary condition. 

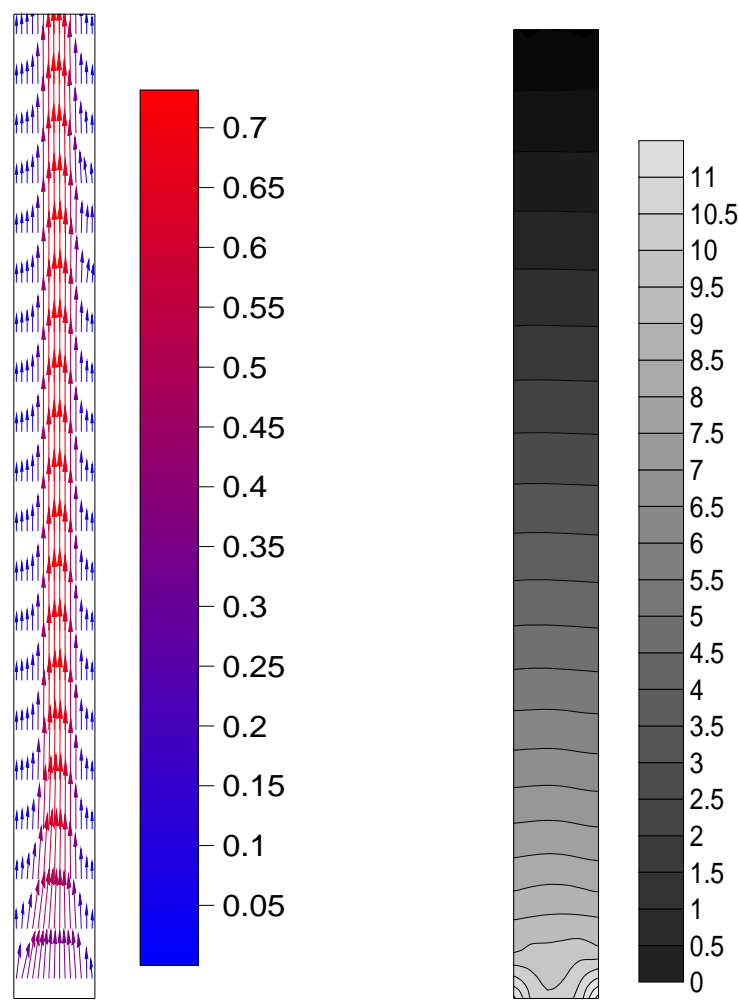

Figure 9. Simulated flow field and pressure distribution within a single $1 \mu \mathrm{m}$ pore after 0.075 seconds with an inlet pressure of $11.5 \mathrm{kPa}$. 


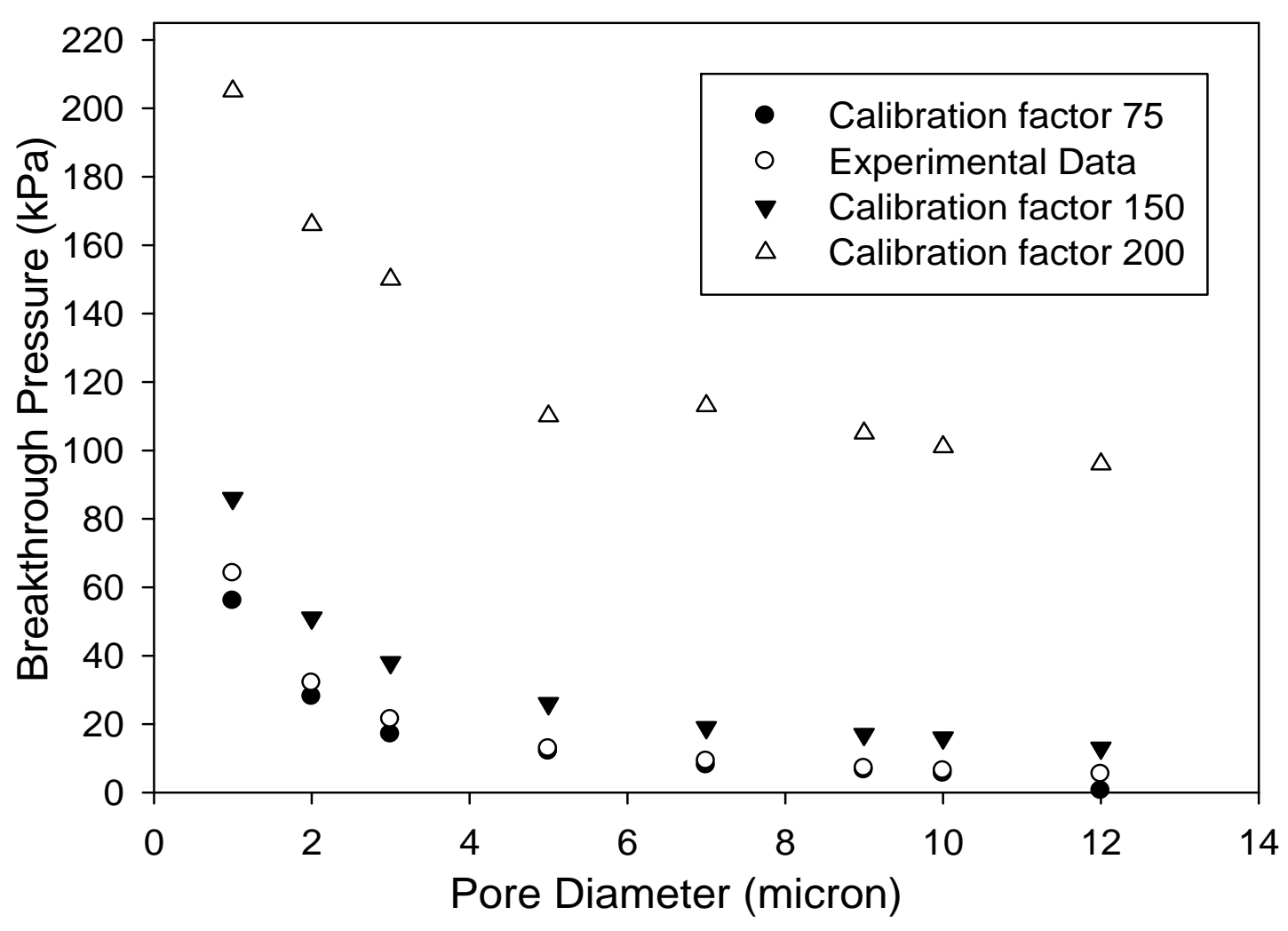

Figure 10. Comparison of computed breakthrough pressures with experimental values, showing the effect of the calibration factors, for various pore diameters. 